\title{
New-generation filler based on cross-linked carboxymethylcellulose: study of 350 patients with 3-year follow-up
}

\author{
Mauro Leonardis' \\ Andrea Palange ${ }^{2}$ \\ 'Department of Plastic Surgery, \\ Salvator Mundi International Hospital, \\ ${ }^{2}$ Department of Aesthetic Medicine, \\ Fisiobios Clinic, Rome, Italy
}

This article was published in the following Dove Press journal:

Clinical Interventions in Aging

6 January 2015

Number of times this article has been viewed

\begin{abstract}
Purpose: In recent years there has been a growing interest in nonsurgical procedures for facial rejuvenation. Hyaluronic acid is currently the most widely used dermal filler for the treatment of facial wrinkles. However, new products with interesting features are being introduced into the market. Cross-linked carboxymethylcellulose is one of these and represents a new alternative for the correction of wrinkles and facial defects.

Patients and methods: The retrospective, multicenter, open-label study on nasolabial folds reported here was carried out between January 2010 and April 2014 on 350 subjects between 22 and 67 years of age for a 36-month follow-up period in order to consistently and extensively assess the safety and performance of this treatment.

Results: The study revealed effective and durable correction of nasolabial wrinkles for periods of 9-12 months. Product reapplication over a 36-month period did not lead to an increase in adverse effects, which always remained rare and of little clinical significance, usually consisting of bruising and redness.
\end{abstract}

Conclusion: Cross-linked carboxymethyl cellulose has been shown to be a safe and effective alternative to the resorbable products currently on the market.

Keywords: dermal fillers, nasolabial fold, hyaluronic acids, facial aging, patient satisfaction

\section{Introduction}

The correction of lines and wrinkles and the restoration of facial shape alterations are key approaches to fight the appearance of aging and enhance facial appearance. In recent years, purified fractions of hyaluronic acid (HA) in the form of chemically stabilized injectable gel have become the standard for nonsurgical correction of facial wrinkles, receiving high patient satisfaction scores. Dermal fillers containing native HA polymer chains alone have little therapeutic utility due to the rapid degradation that occurs within the tissue. In order to obtain acceptable durability in the body, it is essential to perform a chemical process called "cross-linking", which aims to improve in vivo resistance to degradation. ${ }^{1,2}$ According to manufacturers, the chemically cross-linked absorbable HA products available on the market today are classified as "monophasic" or "biphasic": the former come in the form of a homogeneous gel, and the latter consist of particles suspended in solutions used as a carrier (native HA or saline solution). ${ }^{3,4}$

The ideal filler must be biocompatible, nonallergenic, nonmigratory, and must provide long-lasting and reversible effects. Nowadays, product development is focused on improving the aesthetic outcome in terms of softness, a "natural" look and feel of the product in situ, in addition to longevity of results. Consequently, alternative and/or complementary products have been made available on the market today to potentially 
meet these requirements. Carboxymethylcellulose (CMC) is one of these products. Used in the pharmaceutical industry since the $1960 \mathrm{~s}^{5}$ as an excipient and for drug delivery, ${ }^{6-8}$ chemically cross-linked CMC is now available for the correction of facial defects.

\section{Product characteristics}

A commercially available product based on cross-linked CMC (Erelle ${ }^{\mathrm{TM}}$ Total Action, Bioitech Italy Ltd, Rome, Italy) was used for this study. It consists of a non-particulate, viscoelastic, monophasic gel based on cross-linked CMC in isotonic saline solution buffered at $\mathrm{pH} 7$, specially developed for the treatment of facial defects and imperfections.

In biocompatibility testing, cross-linked CMC was found to be noncytotoxic, nonirritant, non-sensitizing, and nongenotoxic. The chemical 1,4-Butanediol diglycidyl ether (BDDE) was selected and used for chemical cross-linking based on 15 years of clinical experience of use in cross-linked $\mathrm{HA},{ }^{1,2}$ resulting in a finished gel with BDDE residue of less than $1 \mathrm{ppm}$.

CMC is synthetically derived from plant sources and presents no protein residues or bacterial endotoxins as compared with HAs, is well tolerated, has a low index of local inflammatory reaction, does not require sensitivity testing, and offers immediate satisfactory effects and acceptable longevity. The rheological characteristics, measured at QI Laboratories Ltd (Pomezia, Italy), were found to be highly efficient. The elastic modulus (G') of approximately $320 \mathrm{~Pa}$ is currently superior to that of all monophasic products on the market, ${ }^{9}$ giving the gel the ability to reside within tissues for long periods of time. The complex modulus $\left(\mathrm{G}^{*}\right)$ was also high, confirming that $\mathrm{CMC}$ has greater ability to withstand skin tension forces and provide better lift and support. In addition, thanks to its high viscosity, the gel presents an optimal ratio between injected amounts and product effects.

\section{Materials and methods Study design}

The retrospective, multicenter, open-label study reported here was conducted at two study sites with two satellite sites (two clinics and two private Italian practices) by two medical researchers, in accordance with the code of good medical practice (GMC). The study was performed on 350 subjects whose first baseline visit (Month 0) was conducted between January 2010 and April 2011. All subjects were followed for a 36-month period. The study was completed in April 2014. The aim of this study was to demonstrate the longterm safety and efficacy of injectable cross-linked CMC gel for the treatment of wrinkles and facial defects. The study was approved by a local ethics review committee internal to participating centers. The research included a review of the clinical files stored in the archives of participating centers. All subjects signed written informed consent and release forms permitting the taking and subsequent use of photographs before the start of any procedure. All subjects enrolled in this study were contacted to sign the authorization form to use their personal data.

Only patients with significant or severe nasolabial folds (NLFs) were included in this study. Folds were visible and symmetrical with a minimum score of 3 on a five-point scale, as per the validated Wrinkle Severity Rating Scale ${ }^{10,11}$ (WSRS is five-point validate scale ranges from "none" [0] to "severe" [5].) This score was reported on the patient's clinical file at baseline (Month 0) before each treatment. On each visit for treatment, the researchers also examined the aesthetic improvement of the subject's NLFs from their pretreatment state using the Global Aesthetic Improvement Scale $^{11}$ (GAIS) (the GAIS is a five-point Likert scale ranging from "much improvement" to "much worse"). An improvement of one grade or more on the WSRS or GAIS from the baseline (Month 0) was considered clinically significant and the subject considered to be a "responder". Statistical significance was calculated using a two-sided binomial test at a 0.05 significance level against a null hypothesis of 0 . Since the purpose of this study was to evaluate the safety and efficacy of the product during its 36-month performance, which also included product reapplication, all subjects were included in the denominator as they were found to be responders at each visit regardless of whether they repeated treatment or not. A further calculation of the average WSRS grade obtained at each session was carried out in order to determine the level of improvement as a function of time and volume of administered product compared with baseline.

The subjects also indicated their degree of satisfaction with the treatment by means of an analogue three-point scale that comprised "Dissatisfied", "Can be improved", and "Satisfied". These ratings were recorded on the patient's clinical file at each visit.

Assessment of treatment outcome using WSRS, GAIS, and degree of satisfaction scales during clinical visits was always carried out before the evaluation of patient retreatment.

Evaluation of the product's safety and tolerability was based on patient reports of adverse events during visits or by telephone, at the time of treatment. These events were always verified by researchers, who directly assessed the reports of 
adverse reactions during scheduled clinical visits, reporting comments on clinical files.

\section{Subjects}

Participants admitted to this study were all aged 18 and over, with a mean age of 47 (range 22-67) years old, having significant or severe NLF of grade 3-5, as defined by the WSRS.

Nonadmission criteria for this study were any pre-existing conditions or conditions that occurred later during the evaluation period that could influence treatment outcome, comprising scars, wounds, filler treatment of the NLFs with other products, previous treatment with permanent or semipermanent facial implants in the treatment area, and significant asymmetry of the NLFs.

During the evaluation period, the use of antiwrinkle treatments in the midface was criterion of nonadmission or exclusion from the study, with the exception of those who had used skincare products for external use.

\section{Treatment}

Eligible treatment sites were the NLFs, in accordance with the validated WSRS protocol.

All subjects received topical anesthetic or injection in preparation for treatment. Cross-linked CMC was applied using a $27 \frac{1}{2}$-gauge needle and application was followed by a massage of the treatment area. Ice was not used before or after treatment. Administration technique (fanning, hatching, anterograde or retrograde administration) and the depth of injection (mid-dermal, subcutaneous, or deep dermis) were at the discretion of the medical researcher.

At baseline (Month 0), the researchers corrected the depth of the fold in each subject as best possible to obtain an improvement of at least one or two grades on the WSRS at the immediate posttreatment evaluation, with consideration of the subject's pretreatment deficiency. If requested, an additional touch-up was administered during the first month, within Week 4. The first posttreatment efficacy evaluation was conducted at Month 3 for all subjects enrolled in the study.

Further treatment of NLFs was not administered before Months 5-7. During this visit, the product was re-administered only if the subject wished retreatment and the WSRS score had worsened by one grade or more from the score documented during the visit at Month 3. Subjects not fulfilling this retreatment criteria at Months 5 and 7 were reassessed using the same criteria between Month 9 and 12, receiving treatment only if the retreatment criteria were met. Maintenance doses were given during the second and third year of follow-up in accordance with the product's longevity in individual subjects, between Months 15 and 18, Months 21 and 24, Months 27 and 30, and Months 33 and 36. Retreatment during the same period of application was not permitted, meaning that a subject who received treatment at Month 5 could not be retreated at Month 6 or 7 but only at the next retreatment period between Month 9 and 12 .

\section{Results \\ Subjects}

A total of 350 subjects met the entry criteria of a WSRS score between 3 and 5. Subjects had a pretreatment score of $3(46.6 \%), 4(42 \%), 5(11.4 \%)$ in line with the depth and visibility of the fold, as documented by medical researchers. The mean age of the subjects was 47 (range 22-67) years, and the majority of the study population was female (82.8\%) and Caucasian (94.8\%).

\section{Dosing and method of administration}

The average amount injected at baseline (Month 0) for the correction of NLFs was $0.6 \mathrm{~mL}$ per side. Within Month 1, $4.8 \%$ of the subjects met the prespecified criteria for supplementary NLF treatment (the average amount administered for touch-up was $0.5 \mathrm{~mL}$ per side). Between Month 5 and 7 , $36.4 \%$ of the study population fulfilled the criteria for retreatment and were retreated (average amount, $0.5 \mathrm{~mL}$ per side). Between Month 9 and 12, 34.5\% of the subjects attending this visit were retreated following confirmation that they fulfilled the protocol-defined criteria (average amount, $0.5 \mathrm{~mL}$ per side). Again, evaluation of treatment outcome, based on the WSRS and GAIS instruments, always occurred prior to assessment for retreatment at these visits. Ten of the subjects treated between Month 5 and 7 (7.9\%) fulfilled criteria for retreatment between Month 9 and 12. Between Month 9 and 12, 29.1\% of the subjects assessed reported still being satisfied with the results and were retreated only at 15 months after the first treatment.

The average evaluation, based on WSRS grade (Figure 1) was significantly improved compared with the average grade at baseline of 3.6 (standard deviation 3-5). These results were maintained for the entire length of the study with the average improvement ranging from 1.2 to $1.9 \mathrm{WSRS}$ grades.

The retrograde tunneling technique was used for $85 \%$ of the subjects, and $80 \%$ of the subjects received injection in the deep dermis. 
Subjects reinjected (\%)

- Cumulative amount reinjected $(\mathrm{mL} / \mathrm{side})$

WSRS mean improvement from baseline (grade)

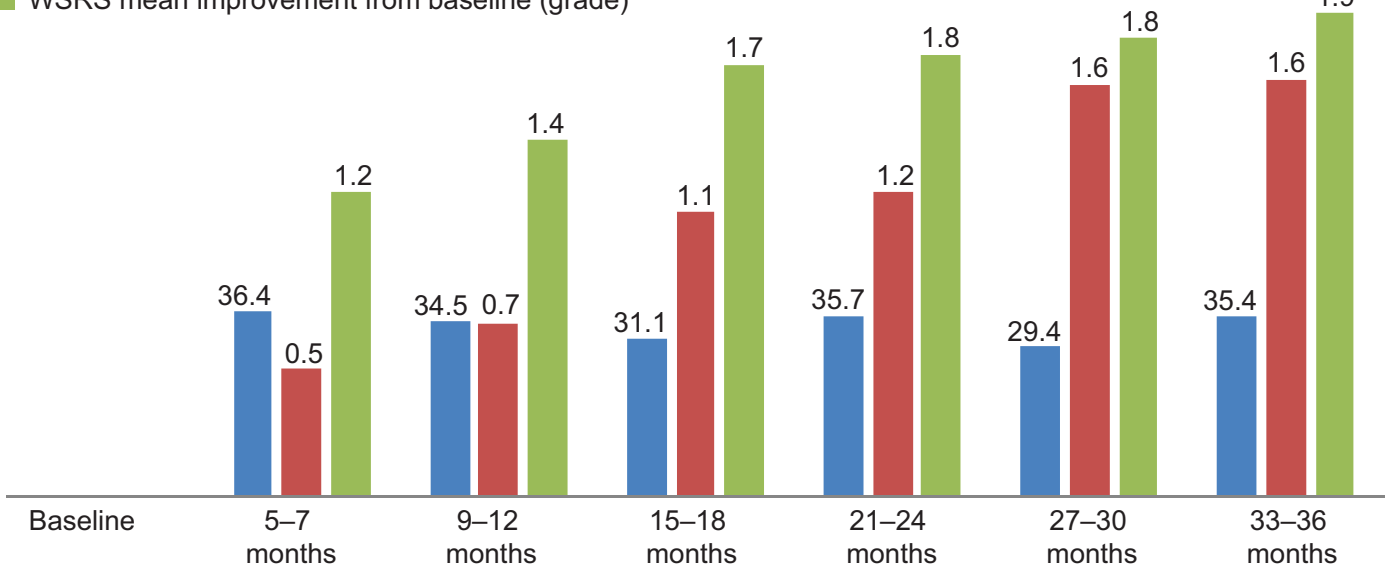

Figure I Percentage of patients receiving treatment as a function of average cumulative volume of injected product per treatment period and corresponding average level of improvement from baseline, as measured by the Wrinkle Severity Rating Scale (WSRS).

\section{Efficacy}

All patient files were reviewed in the time established by the protocol. The percentage of subjects who achieved an improvement of one grade or more on the WSRS compared with baseline (responders) as well as the GAIS responder rate at each clinical visit based on the researcher's assessment are presented in Table 1. Based on this outcome measure, $99.7 \%(P=0.0001)$ of the subjects were "responders" at Month 3 according to the assessments of the medical researchers, thereby demonstrating clinical efficacy. Further treatment of NLFs was not administered before Month 5. The rate of responders as a function of time reveals that nearly two-thirds of the subjects $(63.7 \%)$ had NLF improvement between Month 5 and 7 and about a third (102 [29.1\%]) of them maintained satisfactory results at Month 9 . Seventeen subjects undergoing supplementary treatment within Month 1 were documented as responders at the visit at Month 3 .

Overlapping responses were obtained during the second and third year of product reapplication. A total of 109 subjects (31.1\%) and 125 subjects $(35.7 \%)$ had the product reapplied at Months 15-18 and Months 21-24, respectively, demonstrating a rate of responders equal to about two-thirds of the study subjects. The maximum number of responders $(70 \%)$ was reached during the third year, at Months 27-30.

With regards to subject self-assessment of treatment effects, evaluation always occurred prior to subsequent retreatment. Most (94.8\%) of the subjects reported being "satisfied" at the visit at Month 3. Over two-thirds of the subjects reported being "satisfied" at Months 5-7 and 9-12. At second- and third-year follow-up, satisfaction rates at Months 15-18, 21-24, 27-30, and 33-36 conformed with the trend in the first year, revealing a slight increase in satisfaction, as shown in Figure 2.

The researchers also assessed that $99.7 \%$ were GAIS responders at the visit at Month 3 (Table 1). This result is consistent with the self-assessment made by the subjects. At Months 5-7, the researchers determined that $63.7 \%$ were WSRS and GAIS responders, in line with the $61.1 \%$ of "satisfied" subjects resulting from self-assessment. On subsequent visits and throughout follow-up, this improvement was consistently maintained in both researcher and subject ratings.

Table I Responders according to the Wrinkle Severity Rating Scale (WSRS) and Global Aesthetic Improvement Scale (GAIS) based on the assessment of medical researchers: percentage of subjects maintaining a clinically significant correction of the nasolabial folds during the study phase

\begin{tabular}{|c|c|c|c|c|c|c|c|}
\hline \multirow[t]{2}{*}{ Scale } & \multicolumn{3}{|c|}{ Year I (0-12 months) } & \multicolumn{2}{|c|}{ Year 2 (I 2-24 months) } & \multicolumn{2}{|c|}{ Year 3 (24-36 months) } \\
\hline & 3 months & 5-7 months & $9-12$ months & $15-18$ months & $21-24$ months & 27-30 months & 33-36 months \\
\hline WSRS & 349 (99.7\%) & $223(63.7 \%)$ & $219(62.6 \%)$ & $24 I(68.9 \%)$ & 225 (64.3\%) & 247 (70.6\%) & $226(64.6 \%)$ \\
\hline GAIS & 349 (99.7\%) & $223(63.7 \%)$ & $217(62.0 \%)$ & $238(68.0 \%)$ & $223(63.7 \%)$ & $243(69.4 \%)$ & 221 (63.1\%) \\
\hline
\end{tabular}




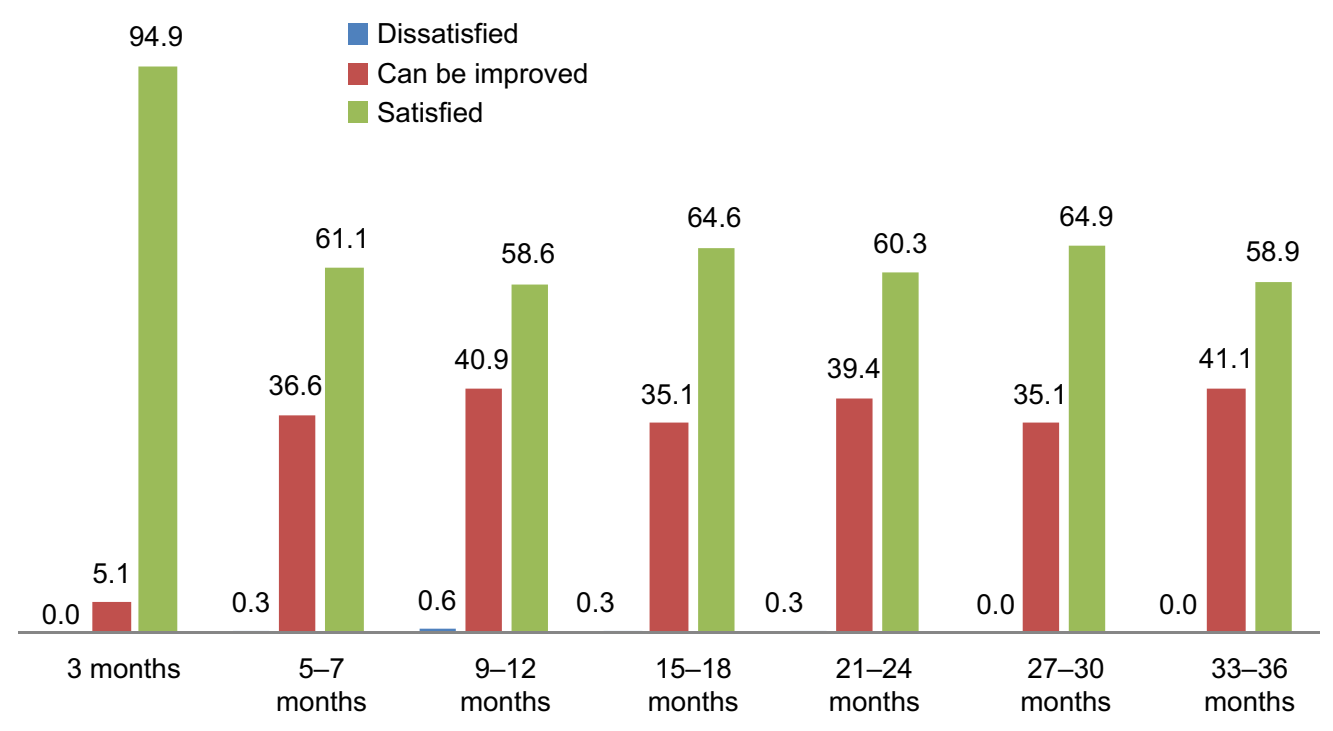

Figure 2 Degree of satisfaction of treatment effects according to study subjects' self-assessment (\%).

The average number of injections per year showed a decrease in reapplication in the second and third years. Even the average amount of injected product decreased with applications, as shown in Table 2 .

\section{Safety and tolerability}

The study treatment was well tolerated, with the majority of treatment-related adverse events being transient, of mild severity, localized to the injection site, and principally consisting of bruising and redness. The post-application pain and tenderness reported by some subjects was always mild and of short duration. The total number of reactions at the injection site related to treatment are presented in Table 3, while the duration and intensity of the adverse effects are reported in Table 4.

There were 147 adverse events during the 36-month study period related to product injection. The most frequent was post-infiltration redness $(16 \%)$. Nodules and persistent erythema at the injection site occurred at very low rates (1.4\%). About two-thirds of adverse events were mild and three-quarters of these resolved within 1 week without sequel $(99.3 \%)$ and did not require treatment $(97.9 \%)$. There were no severe events related to treatment.

\section{Discussion}

"CMC" is a biosynthetic substance based on the b-(1-4)D-glucopyranose polymer of cellulose and is not derived from animal or human sources. ${ }^{12}$ It has been used in the pharmaceutical industry since the $1960 \mathrm{~s}^{5}$ as a carrier for antibiotics, oral anti-inflammatories (ampicillin/ibuprofen), corticosteroid injections (dexamethasone), and for drug delivery (nifedipine/ibuprofen lysine). It is already present in its native state in some dermal fillers with carriers associated with other polymers (eg, Radiesse ${ }^{\circledR}$, Merz Aesthetics, Frankfurt am Main, Germany; EllanséTM, Aqtis Medical, Utrecht, The Netherlands). Cross-linked CMC mixed with cross-linked HA is present on the market as an anti-adhesion surgical product (Seprafilm ${ }^{\circledR}$, Sanofi, Paris, France) that uses a different cross-linking agent known as "carbodiimide" (EDC). Compared with HA, CMC has the advantage of not having protein residues and bacterial endotoxins due to its biosynthetic nature. Furthermore, the pseudoplastic and gel-like features of native $\mathrm{CMC}$ are superior to those present in native HA. This makes it more suitable as a filling and viscosupplementation agent. ${ }^{9}$

The study reported here details the experience of two medical researchers involved from the onset in the study design, development, and marketing of a commercially available injectable gel based on cross-linked carboxymethylcellulose for aesthetic use. The study is a continuation of a preliminary study carried out by the authors in $2010^{13}$ made possible by a protocol determined at the beginning of the experimental phase of cross-linked CMC for aesthetic use that was carried out on a daily basis by the medical researchers in their clinical practice. The aim of the study was to confirm the long-term safety and efficacy of cross-linked

Table 2 Average number of applications per year and average amount of injected product

\begin{tabular}{llll}
\hline Applications & I year & 2 year & 3 year \\
\hline Average number per year & $\mathrm{I} .2 \mathrm{I}$ & $\mathrm{I} .10$ & 0.94 \\
Average amount, $\mathrm{mL} / \mathrm{side}$ & 0.54 & $0.5 \mathrm{I}$ & 0.42 \\
\hline
\end{tabular}


Table 3 Number and distribution of adverse effects in those who suffered from them during the study's 36-month period

\begin{tabular}{lllll}
\hline Event & Adverse events, $\mathbf{n}(\%)$ & \multicolumn{3}{l}{ Distribution in time per adverse event, $\mathbf{n}(\%)$} \\
\cline { 3 - 5 } & & $\mathbf{0 - 1 2 \text { months }}$ & I 2-24 months & 24-36 months \\
\hline Bruising & $23(6.6)$ & $14(60.8)$ & $5(21.7)$ & $4(17.4)$ \\
Swelling/Edema & $3(0.8)$ & $2(66.6)$ & $0(0.0)$ & $1(33.3)$ \\
Pain/Tenderness & $55(15.7)$ & $32(58.2)$ & $11(20.0)$ & $12(21.8)$ \\
Redness & $56(16.0)$ & $33(58.9)$ & $10(17.9)$ & $13(23.2)$ \\
Erythema/Inflammation & $5(1.4)$ & $2(40.0)$ & $1(20.0)$ & $2(40.0)$ \\
Nodules & $5(1.4)$ & $3(60.0)$ & $1(20.0)$ & $1(20.0)$ \\
\hline
\end{tabular}

Note: Total number of study participants: 350 .

CMC in achieving a clinically significant correction of the NLFs and also to assess the duration of the correction during the 36-month study observation period.

The WSRS and GAIS scales ${ }^{12}$ were employed in this study because they are internationally recognized validated research methods of proven reliability. These scales are, by their nature, subjective instruments. Nevertheless, they are a practical tool for the continuous tracking of treatment outcomes and have been used as the primary efficacy parameter in similar studies published in the peer-reviewed literature. ${ }^{4,14}$ The choice to treat WSRS-grade 3-5 folds was made as it would indisputably document the difference between before and after treatment states, as well as test the filling capacity of cross-linked CMC.

Subjects of young age with hereditary genetic defects of the nasolabial area also took part in this study.

Although associated treatments were not considered in this study, they were nevertheless permitted as long as they did not invalidate results. Subjects who had received treatment in the zygomatic region or in the region below the zygomatic before or during the study period were not admitted. Any data on subjects who had received any treatment that could contribute to lifting the buccal region and NLFs, thereby distorting study results, were discarded.

The treatment effects during the first year lasting for up to $9-12$ months and, in $29.1 \%$, up to 15 months (Figures 3 and 4) after one or two treatment sessions were probably related to the physico-chemical and structural properties of cross-linked CMC, which give it better uplift and support and greater resistance to biological degradation deriving from its nonenzymatic-type resorption mechanism. ${ }^{15}$

In the successive two-year study period, the injection amount and the reduction in the number of retreatments compared with the first year confirm the product's durability for periods averaging 9-12 months. These data, together with the increased proportion of responders in the second and third year of application, confirm the product's cumulative effects, also present with the use of other products based on absorbable polysaccharides ${ }^{16}$ indicating the gel's ability to seamlessly integrate into tissues.

Another fact that clearly emerges from this study is that cross-linked CMC is high performing considering that the average amount injected to obtain an effective correction of the folds during the study was lower than the amount generally needed for products based on other polymers. ${ }^{17}$

Observed adverse events suggest that the treatment was well tolerated. Product reapplication during the 36-month study period did not result in an increased incidence of adverse effects. Bruising and swelling were significantly less than expected and those observed in other studies of competing products based on HA, ${ }^{18-20}$ to indicate possible weak hemostatic and anti-edemigenic properties of CMC when infiltrated in the dermis and subcutaneous tissue. Further studies in this direction are needed to confirm this observation.

Anesthesia injection was required in less than $1 \%$ of subjects. It is believed that the gel is less painful to inject as

Table 4 Intensity and duration of adverse events in those who suffered from them

\begin{tabular}{|c|c|c|c|c|c|}
\hline \multirow[t]{2}{*}{ Event } & \multicolumn{3}{|c|}{ Intensity of adverse event, $\mathbf{n}(\%)$} & \multicolumn{2}{|c|}{ Duration of adverse event, $n$ (\%) } \\
\hline & Slight & Moderate & Severe & $<7$ days & $>7$ days \\
\hline Bruising & $14(60.9)$ & $7(30.4)$ & $2(8.7)$ & $14(60.9)$ & $9(39.1)$ \\
\hline Swelling/Edema & $2(66.6)$ & I (33.3) & $0(0.0)$ & $3(100.0)$ & $0(0.0)$ \\
\hline Pain/Tenderness & $33(60.0)$ & $22(40.0)$ & $0(0.0)$ & $55(100.0)$ & $0(0.0)$ \\
\hline Redness & $33(58.9)$ & $21(37.5)$ & $2(3.6)$ & $54(96.4)$ & $2(3.6)$ \\
\hline Erythema/Inflammation & $3(60.0)$ & I (20.0) & I (20) & $3(60.0)$ & $2(40.0)$ \\
\hline Nodules & $2(40.0)$ & $2(40.0)$ & $\mathrm{I}(20)$ & $4(66.6)$ & I (33.3) \\
\hline
\end{tabular}

Note: Total number of study participants: 350 

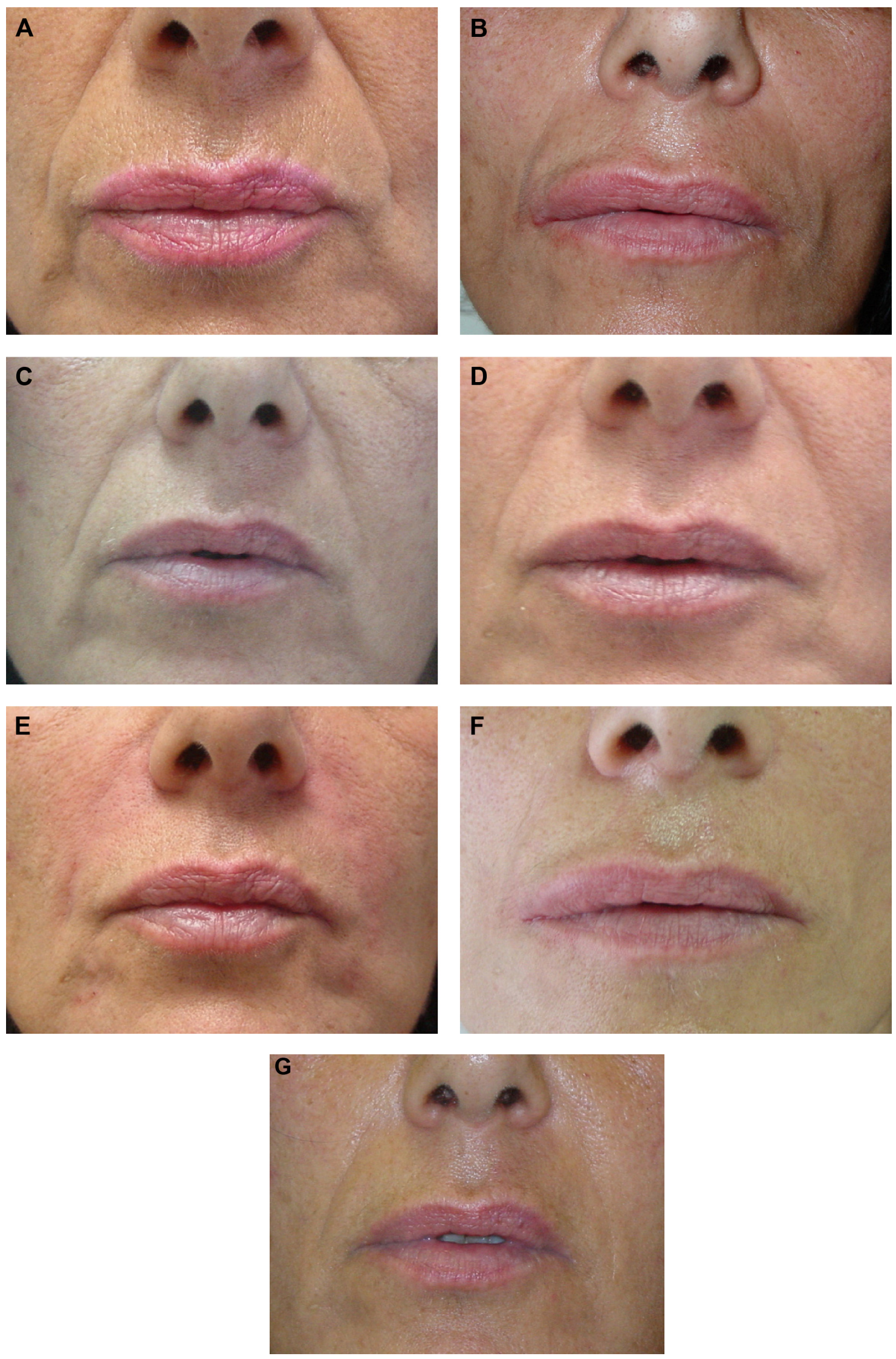

Figure 3 A 48-year-old female (Wrinkle Severity Rating Scale grade 3) before and after application of $0.6 \mathrm{~mL} /$ side of cross-linked carboxymethyl cellulose and reapplication of $0.5 \mathrm{~mL} / \mathrm{side}$ at Month 9.

Notes: (A) Before treatment; (B) Month 3; (C) Month 6; (D) Month 9 before reapplication; (E) Month 9 after reapplication; (F) Month I2; (G) Month I8. 

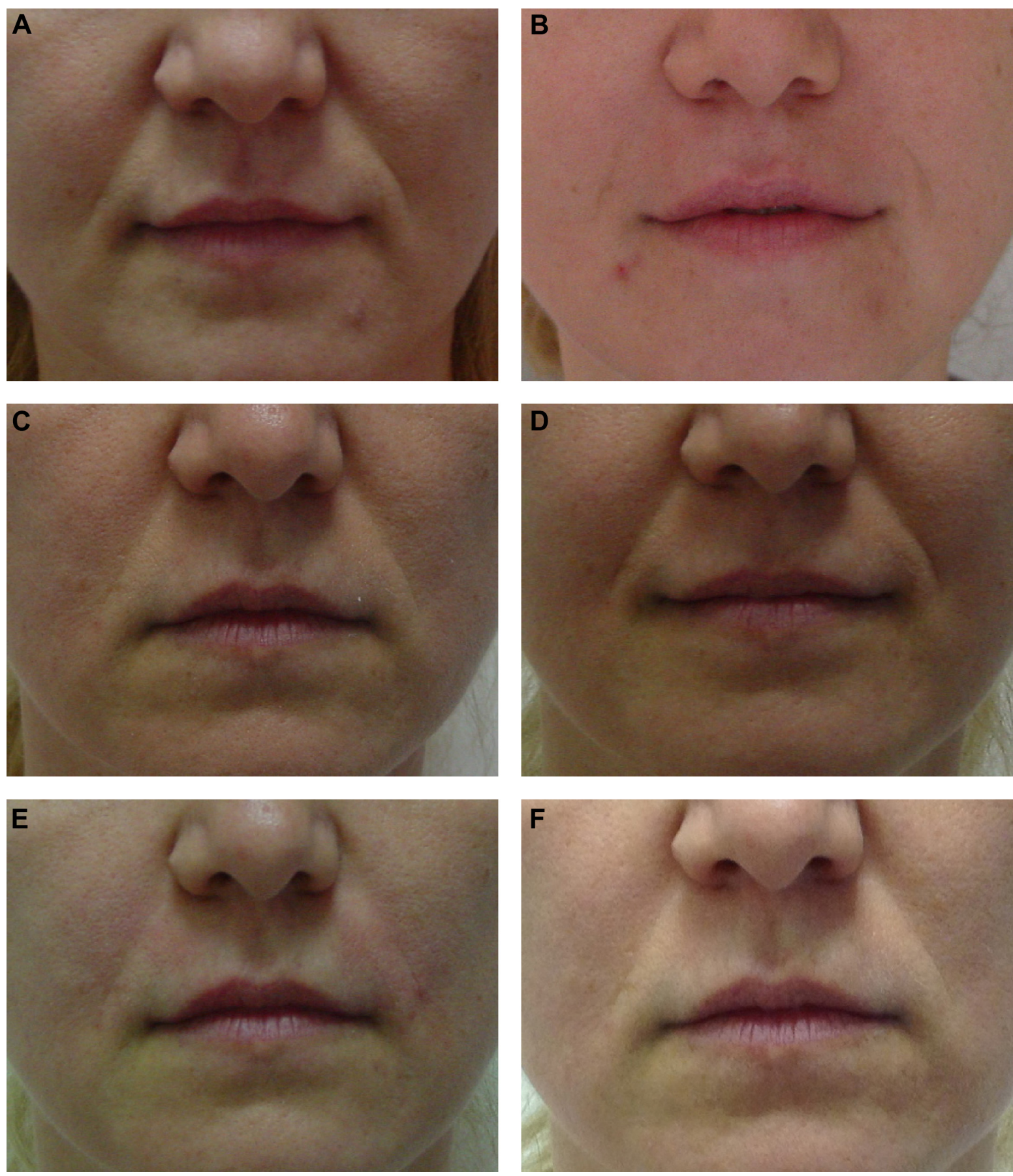

Figure 4 A 38-year-old female (Wrinkle Severity Rating Scale grade 4) before and after application of $0.8 \mathrm{~mL} /$ side of cross-linked carboxymethyl cellulose and reapplication of $0.6 \mathrm{~mL} / \mathrm{side}$ at Month 9 .

Notes: (A) Before treatment; (B) Month 3; (C) Month 6; (D) Month 9 before reapplication; (E) Month 9 after reapplication; (F) Month I5.

compared with other products on the market, making the need for anesthetic injection negligible or providing the possibility of adding lidocaine into the product.

\section{Conclusion}

As far as we are aware, this 36-month retrospective, multicenter, open-label study of 350 subjects is one of the largest and longest of its kind to assess the safety and durability of a resorbable filler based on polysaccharides for the correction of fine lines and facial wrinkles. In this study, in which a majority of subjects had a significant wrinkle depth and visibility, administering treatment with the study product was sufficient to achieve a clinically significant correction in $99.7 \%$ of subjects when evaluated at the 3-month 
posttreatment visit using the WSRS and GAIS. This clinically relevant improvement was maintained in each subsequent evaluation period in about two-thirds of the subjects based on the same rating scales, while the outcome was individually enduring for up to 9-12 months before each subsequent product reapplication without any further treatment during this time interval.

There was a high degree of consistency in the documentation of a clinically meaningful correction during the study, as measured independently by the researchers who used the WSRS and GAIS instruments and the subjects who indicated a corresponding level of satisfaction with the treatment outcome.

Very few adverse effects were reported and these resolved spontaneously in the vast majority of cases within 7 days from the onset without treatment or sequel.

Cross-linked CMC has ultimately proven to be a safe and effective alternative to the resorbable products currently on the market.

\section{Disclosure}

M Leonardis works with Bioitech Ltd on Erelle soft fill, Erelle refinement, Erelle total action and Erelle volume clinical trials. This scientific work is not subsidized or sponsored and has no commercial purpose. The authors report no conflicts of interest in this work.

\section{References}

1. Kablik J, Monheit GD, Yu L, Chang G, Gershkovich J. Comparative physical properties of hyaluronic acid dermal fillers. Dermatol Surg. 2009;35(Suppl 1):302-312.

2. Micheels P, Besse S, Flynn TC, Sarazin D, Elbaz Y. Superficial dermal injection of hyaluronic acid soft tissue fillers: comparative ultrasound study. Dermatol Surg. 2012;38(7 Pt 2):1162-1169.

3. Park KY, Kim HK, Kim BJ. Comparative study of hyaluronic acid fillers by in vitro and in vivo testing. J Eur Acad Dermatol Venereol. 2014;28(5):565-568.

4. Goodman GJ, Bekhor P, Rich M, Rosen RH, Halstead MB, Rogers JD. A comparison of the efficacy, safety, and longevity of two different hyaluronic acid dermal fillers in the treatment of severe nasolabial folds: a multicenter, prospective, randomized, controlled, single-blind, within-subject study. Clin Cosmet Investig Dermatol. 2011;4: 197-205.
5. Courtois, J.E., Bui Khac Diep. Research on the determination of cellulases and hemicellulases in pharmaceutical preparations. II. Preparation and analytical inspection of carboxymethylcellulose. Ann Pharm Fr. 1965;23(11):649-662.

6. Barbucci R, Leone G, Vecchiullo A. Novel carboxymethylcellulosebased microporous hydrogels suitable for drug delivery. J Biomater $S_{c i}$ Polym Ed. 2004;15(5):607-619.

7. Sangalli ME, Giunchedi P, Colombo P, Conte U, Gazzaniga A, La Manna A. Cross-linked sodium carboxymethylcellulose as a carrier for dissolution rate improvement of drugs. Boll Chim Farm. 1989;128(7-8): 242-247.

8. Mohammed FA. Topical permeation characteristics of diclofenac sodium from NaCMC gels in comparison with conventional gel formulations. Drug Dev Ind Pharm. 2001;27(10):1083-1097.

9. Falcone SJ, Doerfler AM, Berg RA. Novel synthetic dermal fillers based on sodium carboxymethylcellulose: comparison with Crosslinked hyaluronic acid-based dermal fillers. Dermatol Surg. 2007;33 Suppl 2:S136-S143.

10. Day DJ, Littler CM, Swift RW, Gottlieb S. The wrinkle severity rating scale: a validation study. Am J Clin Dermatol. 2004;5(1):49-52.

11. Narins RS, Brandt F, Leyden J, Lorenc ZP, Rubin M and Smith S. A randomized, double-blind, multicenter comparison of the efficacy and tolerability of Restylane versus Zyplast for the correction of nasolabial folds. Dermatol Surg. 2003;29:588-595.

12. Gruzman MK, Bashura GS. Rheolocic properties of sodium-carboxymethyl cellulose and methylcellulose. Farm Zh. 1964;19: $53-58$.

13. Leonardis M, Palange A, Dornelles RF, Hund F. Use of cross-linked carboxymethyl cellulose for soft-tissue augmentation: preliminary clinical studies. Clin Interv Aging. 2010;9;5:317-322.

14. Ascher B, Bayerl C, Brun P, et al. Efficacy and safety of a new hyaluronic acid dermal filler in the treatment of severe nasolabial lines - 6-month interim results of a randomized, evaluator-blinded, intra-individual comparison study. J Cosmet Dermatol. 2011;10:94-98.

15. Gottfried Lemperle et al. Human Histology and Persistence of Various Injectable Filler Substances for Soft Tissue Augmentation Aesth. Plast Surg. 2003;27:354-366.

16. Narins RS, Dayan SH, Brandt FS, Baldwin EK. Persistence and improvement of nasolabial fold correction with nonanimal-stabilized hyaluronic acid 100,000 gel particles/mL filler on two retreatment schedules: results up to 18 months on two retreatment schedules. Dermatol Surg. 2008;34 Suppl 1:S2-S8; discussion S8.

17. Calcium Hydroxylapatite versus Nonanimal Stabilized Hyaluronic Acid for the Correction of Nasolabial Folds: A 12-Month, Multicenter, Prospective, Randomized, Controlled, Split-Face Trial. Moers-Carpi MM, Tufet JO. Dermatol Surg. 2008;34(2):210-215.

18. Duranti F.et al. Injectable Hyaluronic acid gel for soft tissue augmentation. Dermatol. Surg. 1998;24:1317-1325.

19. Carruthers A. et al. "Randomized, Double blind comparison of the efficacity of two hyaluronic acid derivatives, Restylane Perlane and hylaform, in the treatment of nasolabial fold." Dermatolog Surg. 2005;31: 1591-1598.

20. Lowe NJ. et al. "Hyaluronic acid skin fillers: adverse reactions and skin testing." J Am Acad Dermatol. 2001;45:930-933.
Clinical Interventions in Aging

\section{Publish your work in this journal}

Clinical Interventions in Aging is an international, peer-reviewed journal focusing on evidence-based reports on the value or lack thereof of treatments intended to prevent or delay the onset of maladaptive correlates of aging in human beings. This journal is indexed on PubMed Central, MedLine,

\section{Dovepress}

CAS, Scopus and the Elsevier Bibliographic databases. The manuscript management system is completely online and includes a very quick and fair peer-review system, which is all easy to use. Visit http://www.dovepress. com/testimonials.php to read real quotes from published authors. 